\title{
Production of Recombinant Retroviruses Encoding Human Flt3 Ligand and IL-6 and Establishment of Genetically Modified OP9 Mouse Stromal Cells
}

\author{
Ho-Bum Kang ${ }^{1,2}$, Young-Eun Kim¹, Hyo-Jin Kang ${ }^{1}$, Ae-Ran Lee ${ }^{1}$, \\ Dai-Eun Seok ${ }^{2}$ and Younghee Lee $^{*}$ \\ ${ }^{1}$ Department of Biochemistry, College of Natural Sciences, Chungbuk National University, \\ Cheongju, Chungbuk, 361-763, Korea and ${ }^{2}$ Department of Pharmacy, College of Pharmacy, \\ Chungnam National University, Yusong, Daejon, 305-764, Korea
}

Received : December 2, 2006

Accepted : January 24, 2007

\begin{abstract}
Flt3 Ligand (FL) and IL-6 are multifunctional cytokines implicated in normal hematopoiesis and ex vivo expansion of hematopoietic stem cells. Retroviral vectors are useful for stable expression of genes in many cells. Here, we aimed to produce retroviral vectors directing expression of human FL and IL-6 genes. Recombinant retroviral vectors containing human genes for FL and IL-6 were constructed using a retroviral vector pLXSN. Recombinant retroviruses were produced from GP2-293 cells with the aid of pseudo-envelope protein gene VSV-G, and efficiently transduced to a mouse stromal cell line OP9. Genetically modified OP9 cells clearly showed expression of human FL or IL-6 gene at the mRNA level determined by RT-PCR. Based on the results from ELISA, human FL and IL-6 were detected in the cell culture medium of OP9/FL and OP9/IL-6 cells, respectively. As the recombinant human FL and IL-6 proteins are successfully produced and secreted to the culture medium, this system can be useful in future application such as ex vivo expansion of hematopoietic stem cells and differentiation of embryonic stem cells.
\end{abstract}

Key Words: Hematopoiesis, Retrovirus, Stromal cell, Flt3 Ligand, IL-6

\section{INTRODUCTION}

Blood cell production is maintained by a balance between self renewal, proliferation, apoptosis, and differentiation of hematopoietic stem/progenitor cells (HSCs), processes regulated in part by cytokine-cell interactions. Hematopoietically relevant cytokines have been identified and their implications were widely studied $(5,6)$. Cytokines such as stem cell factor (SCF), thrombopoietin (TPO), Flt3 ligand (FL), BMP4, and

\footnotetext{
${ }^{*}$ Corresponding author: Younghee Lee, Ph.D. Department of Biochemistry, College of Natural sciences, Chungbuk National University, 48, Gaesin-dong, Cheongju, Chungbuk, 361-763, Korea. Phone: 82-43-261-3387, Fax: 82-43-267-2306 e-mail: yh14177@chungbuk.ac.kr

** This research was supported by a grant (SC2110) from Stem Cell Research Center of the 21st Century Frontier Research Program funded by the Ministry of Science and Technology, Republic of Korea.
}

IL-6 were implicated in self renewal of HSCs in human and mouse $(3,19,22,27,33)$.

FL is a potent cytokine that acts synergistically with a wide range of colony stimulating factors (CSFs) and interleukins to stimulate proliferation and differentiation of hematopoietic stem and progenitor cells (15). It also enhances survival of progenitor cells (30). FL binds to cells expressing the Fms-related tyrosine kinase receptor Flt3. Multiple isoforms of FL have been identified (12). The predominant biologically active form is anchored to the cell surface as an extracellular domain of transmembrane protein consisting 209 amino acid residues, and the soluble active form comprising 155 amino acid residues is generated by proteolytic cleavage of the extracellular domain. FL proteins are conserved in human and mouse with $72 \%$ identity in amino acid sequences, and they have cross-reactivity. 
Interleukin-6 (IL-6) is a multifunctional protein that plays important roles in host defense, acute phase reactions, immune responses, and hematopoiesis (21). IL-6 is expressed by a variety of normal and transformed cells including $\mathrm{T}$ cells, B cells, monocytes/macrophages, fibroblasts, hepatocytes, keratinocytes, astrocytes, vascular endothelial cells, and various tumor cells $(2,13,18)$. The production of IL-6 is up-regulated by numerous signals including mitogenic or antigenic stimulation, LPS, calcium ionophore, IL-1, IL-2, IFN, TNF, PDGF, and viruses (7). In comparison with mouse IL-6, human IL-6 exhibits approximately $65 \%$ and $42 \%$ sequence identity at the nucleotide and the amino acid levels, respectively. Although human and mouse IL-6 are equally active on mouse cells, mouse IL-6 is not active on human cells (11).

Replication incompetent retroviruses derived from murine leukemic virus (MLV) and other retroviruses are popular vectors for gene transfer in many cells including HSCs (8). Gene transfer using adenoviral or adeno-associated viral vector system is also efficient, but the expression of the introduced gene is transient as the recombinant viral genome does not integrate at all or integrate with very low efficiency into the chromosome. Retroviruses stably integrate their genome into actively dividing cells and efficiently express foreign gene products (20).

Here, we produced recombinant retroviral vectors containing human FL or IL-6 genes and established OP9 mouse stromal cells expressing human FL or IL-6. Recombinant retroviruses and genetically modified OP9 cells will be useful in future studies.

\section{MATERIALS AND METHODS}

\section{Construction of recombinant retrovirus for expres- sion of cytokines}

In order to construct recombinant retroviral vectors for expression of human FL and IL-6 genes, pLXSN was used as a retroviral expression vector. cDNAs encoding full length open reading frame for these cytokines were obtained by PCR using Marathon-Ready cDNA from human bone marrow (Clontech, Palo Alto, CA, USA) as a template. 0.1 ng of cDNA was subjected to the standard PCR reaction using Ex Taq polymerase (Takara, Shiga Japan) for 35 cycles of denaturation for $60 \mathrm{sec}$ at $95^{\circ} \mathrm{C}$, annealing for 60 sec at $59^{\circ} \mathrm{C}$, and elongation for $60 \mathrm{sec}$ at $72^{\circ} \mathrm{C}$. The primer sequences used are as follows: for FL (708 bp), 5'-ATGACAGTGCTGGCGCCAGCCTGGA-3' and 5'-TCAGTGCTCCACAAGCAGCAGGTCC-3'; for IL-6 (639 bp), 5'ATGAACTCCTTCTCCACAAGCGCCT-3' and 5'-CTACATTTGCCGAAGAGCCCTCAGG-3'. RT-PCR products were cloned by TA cloning, and confirmed by DNA sequencing and BLAST search. cDNA fragments were digested with Eco RI and Bam HI followed by purification and ligation into pLXSN (Clontech) predigested with the same enzyme set. Ligation mixture was transformed into E. coli strain DH5 $\alpha$, and transformants were selected and confirmed by plasmid preparation and restriction analysis.

\section{Cell culture and retroviruses preparation}

\section{1) Culture of GP2-293 cells}

GP2-293, a cell line derived from human embryonic kidney cell line HEK293, was obtained from Clontech and used as a packaging cell line for preparation of retroviruses. GP2-293 cells were maintained in Dulbecco's modified Eagle's medium (DMEM, Gibco BRL, Grand Island, NY, USA) containing $10 \%$ fetal bovine serum (FBS, Hyclone, Logan, UT, USA) in $5 \% \mathrm{CO}_{2}$ incubator at $37^{\circ} \mathrm{C}$.

\section{2) Transfection of retroviral vectors into GP2-293 cells} GP2-293 cells $\left(2 \times 10^{5}\right.$ cells) were placed in a $10 \mathrm{~cm}$ cell culture dish and cultured for $24 \mathrm{hrs}$. Ten microgram of retroviral vector pLXSN, pLXSN-FL or pLXSN-IL6 was transfected into the cells by calcium-phosphate precipitation method using CalPhos ${ }^{\mathrm{TM}}$ Mammalian transfection Kit (Clontech). After $12 \mathrm{hr}$ incubation, the medium was exchanged with fresh culture medium and cultured for another 48 hrs. Cells were split and selected in a medium containing 1 $\mathrm{mg} / \mathrm{ml}$ of G418. Expression of cytokine genes was verified by RT-PCR using mRNAs from the G418-resistant cells.

\section{3) Harvest of the recombinant retroviruses}

G418-resistant GP2-293 cells ( $3 \times 10^{5}$ cells) were placed in a $10 \mathrm{~cm}$ cell culture dish and cultured for $24 \mathrm{hrs}$. Cells were transfected with pVSV-G plasmid (Clontech) encoding pseudo-envelope protein gene using calcium-phosphate precipitation method. Twelve hrs later, the medium was exchanged with fresh culture medium supplemented with $10 \mathrm{mM}$ sodium butyrate. After $48 \mathrm{hrs}$, the supernatant of the culture medium was taken and filtrated through a filter with a 0.45 $\mu \mathrm{m}$ pore size. The retrovirus supernatants were concentrated 


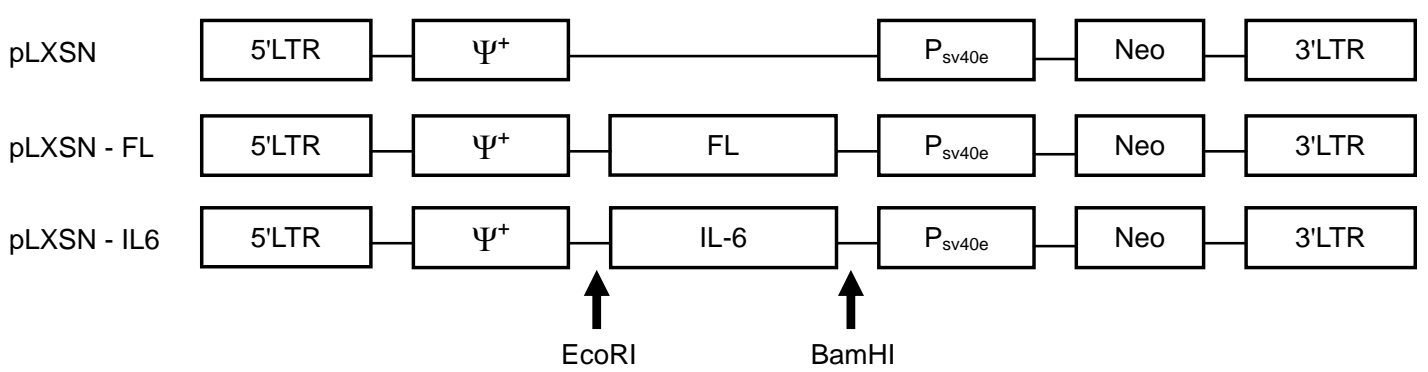

Figure 1. Maps of the retroviral vectors used in this study. pLXSN including LTR promoter and Neo resistant marker was used as a parental cloning vector. Eco RI and Bam HI sites were used for cloning of FL and IL-6.

using Centricon (PL-10, Bedford, MA, USA) and stored at $-80^{\circ} \mathrm{C}$.

\section{4) Titration of the retroviruses}

Titers of the retroviruses were measured by colony formation frequency after infection into a mouse embryonic fibroblast cell line NIH3T3. NIH3T3 cells were obtained from the American Type Culture Collection (ATCC, Manassas, VA, USA) and maintained in DMEM containing 10\% FBS. A day before experiment, NIH3T3 cells were placed in a 6 well plate. One milliliter of viral supernatants serially diluted in the range of $10^{2} \sim 10^{6}$ fold was applied to each well and $8 \mu \mathrm{g} / \mathrm{ml}$ of polybrene was added. Twenty four hrs later, G418 was added at the concentration of $1 \mathrm{mg} / \mathrm{ml}$ and incubated for 2 weeks. When the G418-resistant colonies were visible, the medium was removed and the colony numbers were counted after methylene blue staining.

\section{5) Transduction of retroviruses into OP9 cells}

Biological activity of the recombinant retroviruses was verified by infection of these viruses into a mouse stromal cell line OP9. OP9 cells were placed in a $10 \mathrm{~cm}$ cell culture dish a day before transduction. Fifty MOI of viral supernatant was applied with $8 \mu \mathrm{g} / \mathrm{ml}$ of polybrene. Twenty four hrs later, G418 was added at the concentration of $1 \mathrm{mg} / \mathrm{ml}$ and G418-resistant OP9 cells were selected.

\section{RT-PCR}

Total RNA was isolated using TRI REAGENT ${ }^{\circledR}$ according to the instructions provided by the manufacturer (MRC, Cincinnati, OH, USA). Five microgram of mRNA was reverse-transcribed in the first-strand buffer containing $6 \mu \mathrm{g} /$ $\mathrm{ml}$ oligo(dT) primer, $50 \mathrm{U}^{\text {StrataScript }}{ }^{\mathrm{TM}}$ reverse transcriptase (Stratagene, La Jolla, CA, USA), 2 mM dNTP, and 40 U RNase block ribonuclease inhibitor. One microliter of the cDNA synthesis was subjected to the standard PCR reaction for 35 cycles of denaturation for $60 \mathrm{sec}$ at $95^{\circ} \mathrm{C}$, annealing for $60 \mathrm{sec}$ at $55^{\circ} \mathrm{C}$ and elongation for $60 \mathrm{sec}$ at $72^{\circ} \mathrm{C}$.

\section{Protein assay by ELISA}

To verify the expression and secretion of recombinant cytokines, protein amount in the cell culture medium was measured by sandwich ELISA method. ELISA experiments were performed by Bank for Cytokine Research at Chonbuk National University (Jeonju, Korea) using Quantikine system (R\&D Systems, Minneapolis, MN, USA) according to the manufacturer's recommendation. In brief, a series of 2-fold diluted cytokine solutions were used as a standard. Optical density was measured at $430 \mathrm{~nm}$. Potting standard curves and quantifying cytokine concentration was done by using Sigma Plot.

\section{RESULTS}

\section{Construction of retroviral vectors encoding human}

\section{FL or IL-6 genes}

pLXSN is a retroviral vector and has multicloning sites in which the downstream genes can be cloned and regulated under the control of LTR (long terminal repeat) promoter. A selection marker $\mathrm{NeO}$ gene can be expressed by constitutive promoter SV40. cDNA clones encoding full length open reading frame of human FL (708 bp) and IL-6 (639 bp) cDNA were isolated from human bone marrow, and subcloned into pLXSN to produce pLXSN-FL and pLXSNIL6, respectively. The maps of the retroviral vectors used in this study are presented in Fig. 1. Recombinant retroviral vectors were verified by restriction analysis and PCR with the primers used for cDNA cloning. 
(A)

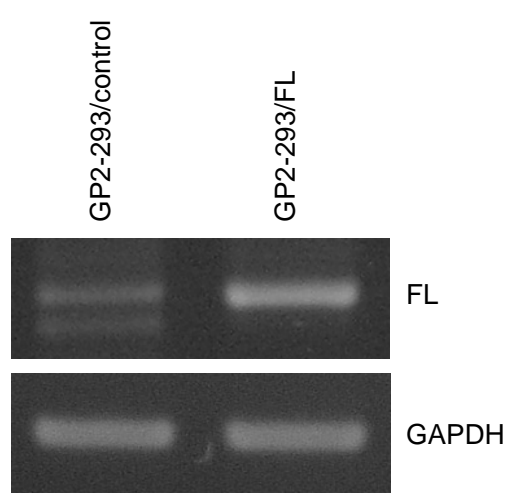

B

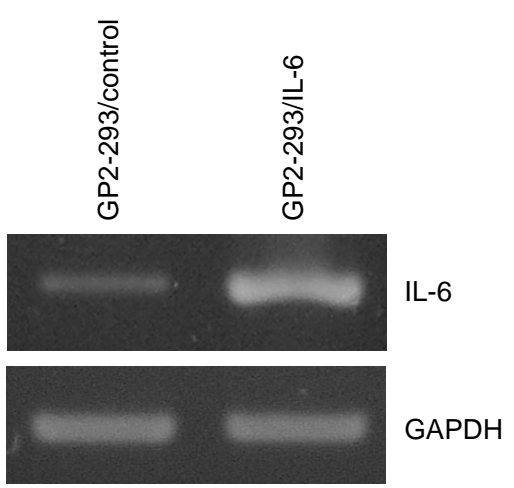

Figure 2. Expression of human FL and IL-6 genes in GP2-293 cells transfected with retroviral vectors. GP2-293 cells were transfected with retroviral vectors and selected in the presence of G418. Total RNAs were prepared from the G418-resistant cells and analyzed by RT-PCR. (A) Expression of human FL mRNA in GP2-293/FL cells. (B) Expression of human IL-6 mRNA in GP2-293/IL-6 cells. Expression of GAPDH was used as a RNA amount control.

\section{Isolation of stable cell pools harboring retroviral vector sequences integrated in the chromosome}

In order to produce replication-incompetent retroviruses, we used a packaging cell line GP2-293. GP2-293 cells have chromosome-integrated gag and pol genes which are essential for retrovirus replication and production of viral particles. GP2-293 cells were transfected with pLXSN, pLXSNFL, or pLXSN-IL6. Tansfection efficiency was about 70 to $80 \%$, which was determined by control experiment using a retroviral vector encoding GFP. As the retroviral vectors have Neo gene, we first selected GP2-293 transfectant cells resistant to G-418 and named as GP2-293/control, GP2293/FL, and GP2-293/IL-6, respectively. Expression of the cloned genes was verified by RT-PCR (Fig. 2). Although GP2-293/contol cells show basal expression of human FL and IL-6, GP2-293/FL and GP2-293/IL-6 cells do express the genes at higher levels compared with GP2-293/control cells. As GP2-293 cells were originated from human embryonic kidney cells, the result is consistent with the information from GeneCards website on the FL and Il-6 gene expression in human tissues analyzed by UniGene electronic Northern (http://www.genecards.org): expression of FL and IL-6 was commonly seen in lung, thymus, pancreas, and kidney.

\section{Production of retroviruses}

GP2-293/control, GP2-293/FL and GP2-293/IL-6 cells were again transfected with pVSV-G vector encoding $G$ glycoprotein of the vesicular somatitis virus. VSV-G is
(A)

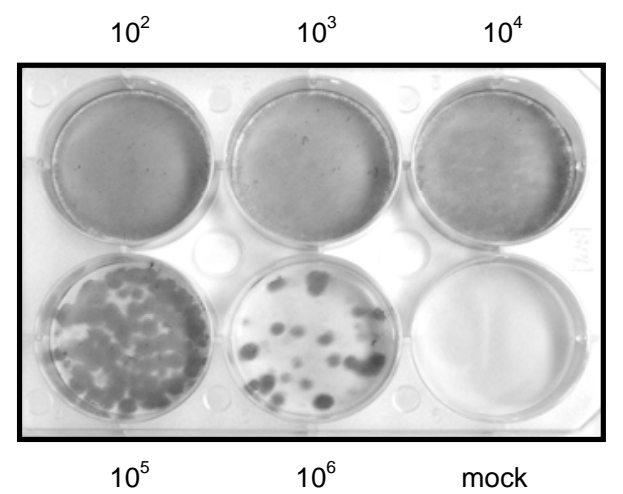

B

\begin{tabular}{|c|c|}
\hline Virus & Titers \\
\hline retrovirus/control & $2 \times 10^{6}$ \\
\hline retrovirus/FL & $6 \times 10^{6}$ \\
\hline retrovirus/IL-6 & $2 \times 10^{6}$ \\
\hline
\end{tabular}

Figure 3. Titration of retroviruses. Viral supernatants of the packaging cells GP2-293/FL and GP2-293/IL-6 were harvested and NIN3T3 cells were transduced with 10-fold serial dilutions of the retroviral supernatant $\left(10^{2}\right.$ to $10^{6}$ dilutions) or untransduced (mock). After two-week culture in the presence of G418, numbers of G418-resistant colonies were counted. (A) Representative result of titration experiments. (B) Calculated titers of the retroviruses.

used in peudotyping of Moloney Murine Leukemia Virus (MMLV)-based retroviral vectors by mediating viral entry. VSV-G interacts with phospholipid of the target cell membrane and fosters fusion of viral and cellular membranes. Culture supernatants including the recombinant retroviruses were harvested and titrated using mouse fibroblast cell line NIH/3T3 cells as an infection host (Fig. 3). The titers of retrovirus/control, retrovirus/FL and retrovirus/IL-6 were 
A

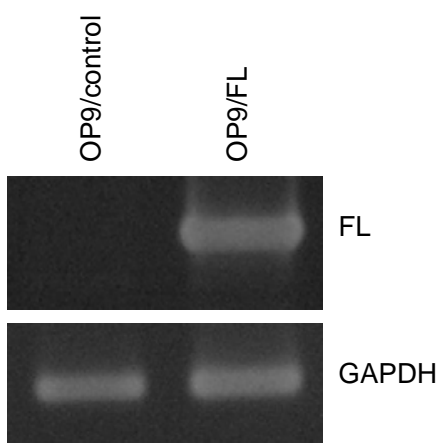

B

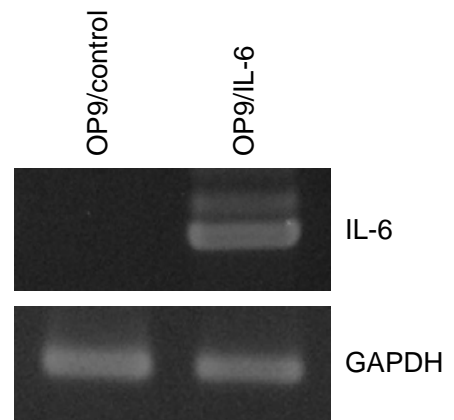

Figure 4. Expression of human FL and IL-6 genes in OP9 cells transduced with retroviruses. OP9 cells were infected by retroviruses and selected in the presence of G418. Total RNAs were prepared from the G418-resistant cells and analyzed by RT-PCR. (A) Expression of human FL mRNA in OP9/FL cells. (B) Expression of human IL-6 mRNA in OP9/IL-6 cells. Expression of GAPDH was used as a RNA amount control.

A

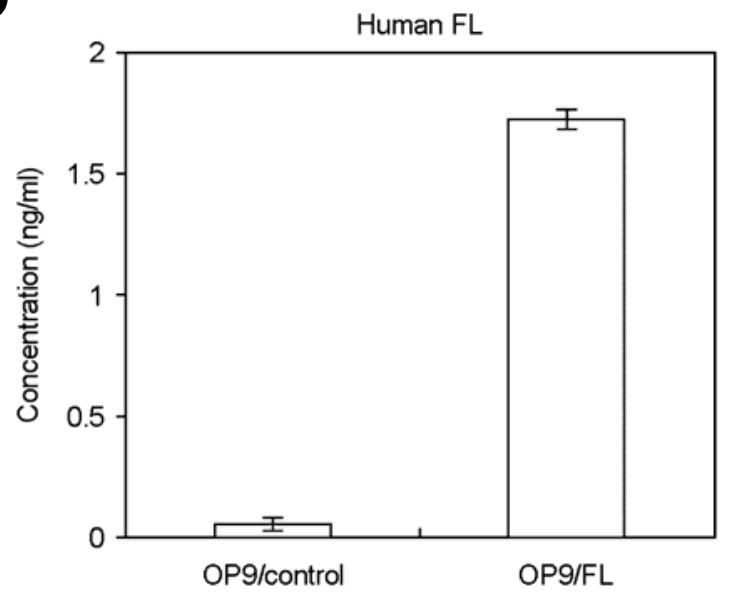

B

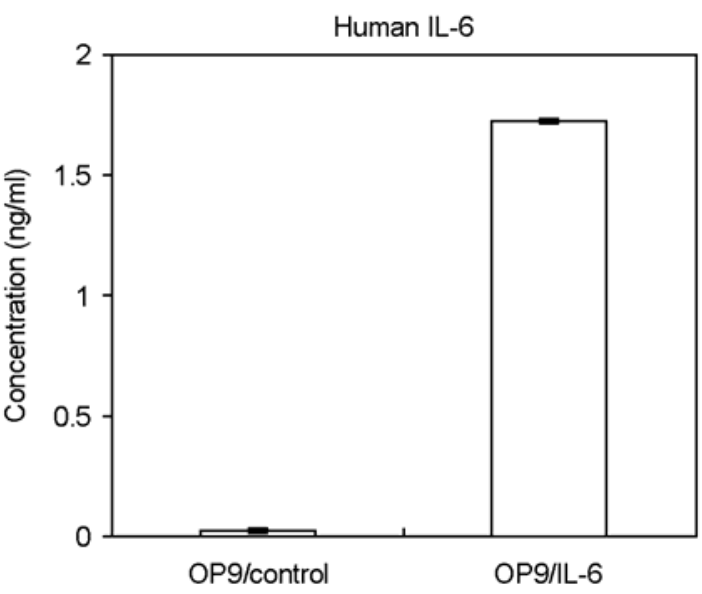

Figure 5. Expression of human FL and IL-6 proteins in the culture media of OP9/FL and OP9/IL6 cells. Culture supernatants of OP9/ control, OP9/FL and OP9/IL6 cells were harvested and the amounts of human FL (A) and IL-6 (B) proteins were measured by ELISA method. Data are presented as a mean value of triplicate experiments.

around $2 \sim 6 \times 10^{7} \mathrm{pfu} / \mathrm{ml}$.

\section{Infection of retroviruses to mouse stromal cell line} OP9

Viral supernatants including retrovirus/control, retrovirus/ FL or retrovirus/IL-6 were allowed to infect mouse stromal cell line OP9 and G418-resistant cells were selected to get OP9/control, OP9/FL and OP9/IL6 stably expressing null, FL, and IL-6, respectively. The expression of human FL and IL-6 mRNAs was verified by RT-PCR (Fig. 4). As expected, there was no PCR product detected in OP9/control cells.

\section{Secretion of cytokines in the culture medium of genetically modified OP9 cells}

As expression of FL and IL-6 was examined in the OP9/
FL and OP9/IL-6 cells at the mRNA level, we decided to check the protein level. When the cytokines are produced in the cells, they are secreted into the medium. Therefore, supernatants of the cell culture were harvested and protein amounts were determined by quantitative ELISA method (Fig. 5). In the culture medium of OP9/control cells, there was no detectable amount of human FL or IL-6. In contrast, human FL and IL-6 were detected in the culture media of OP9/FL and OP9/IL-6 cells, respectively. The concentration was about $1.7 \mathrm{ng} / \mathrm{ml}$ after 1 day culture. When we examined moue FL in the culture medium of OP9/control cells as well as OP9/FL cells, it was not detected by ELISA. According to the manufacturer's manual, the minimum detectable doses of human FL, human IL-6, and mouse FL were $7 \mathrm{pg} / \mathrm{ml}$, $0.7 \mathrm{pg} / \mathrm{ml}$, and $5 \mathrm{pg} / \mathrm{ml}$, respectively. 


\section{DISCUSSION}

Hematopietic stem cells producing all kinds of blood cells are widely used in transplantation in leukemic disease or after cancer therapy. Recently, the finding of plasticity of adult stem cells has also increased the interest in HSCs as a potential therapy for a wide range of nonhematological disorders. Traditionally, bone marrow was the main source of HSCs. However, the advantage of HSC from cord blood (CB) has been widely known. CB has higher frequency of HSCs, and has lower immunological barrier due to the naïveté of the immature $\mathrm{T}$ cell population compared to bone marrow or mobilized peripheral blood. However, the small quantity of $\mathrm{CB}$ is a main limiting factor in the usage of $\mathrm{CB}$, which decreases the success rate of transplantation and restricts transplantation to the treatment of small children (4). As CB is a readily available source of HSCs and there are a number of CB banks worldwide, ex vivo expansion without loss of stem cell properties is an important issue in application of CB HSCs.

For expansion of HSCs, they usually use cytokine cocktails including FL, TPO, IL-6, or SCF which were implicated in self renewal. Here, we focused on FL and IL-6. As human proteins of these cytokines are active in human as well as in mouse, they can be used in both systems. In this study, we constructed retroviral vectors encoding human FL or IL-6 and found these viruses are capable of infecting mouse stromal cell OP9 and directing expression and secretion of human recombinant cytokines in the cells.

Hematopoiesis involves a complex set of interactions that are dynamically and finely regulated by the environment and specifically by stromal cells (23). Stromal cells regulate hematopoiesis by binding directly to hematopoietic stem cells (HSCs) and also by providing numerous secreted cytokines $(9,16,17)$. Therefore, co-culture system is believed to be more efficient in maintaining and expanding HSC population without loss of stem cell activity. As we established stable stromal cell lines expressing FL or IL-6, they will be very useful in future study, for example, expansion of mouse HSCs with either alone or combination.

Human embryonic stem (ES) cell lines have been derived from the inner cell mass of preimplantation embryos by culturing the cells on mouse embryonic fibroblast (MEF) cells $(14,26,28)$. In these conditions, human ES cells show remarkable proliferative capacity and stability in long-term culture (1) and have the capacity to differentiate into cell types from all three germ layers both in vitro and in vivo $(26,28)$. For hematopoietic differentiation from mouse ES cells, they usually use mouse stromal cell line OP9 $(10,24$, 25). Even in the differentiation of human ES cells, they still use mouse OP9 cells $(29,31,32)$. Therefore, our cell lines can be used in this system to enhance the efficiency of differentiation especially in human ES cells. In the case of IL-6, mouse protein is not active in human. As we introduced human IL-6 in OP9 cells (OP9/IL-6), they will be more powerful in terms of IL- 6 production and activity on human cells. FL proteins are conserved in human and mouse and they have cross-reactivity. When we checked the culture medium of OP9/control cells using ELISA system, mouse FL was not detected (data not shown). In contrast, human FL was clearly detected in the culture medium of OP9/FL cells. Taken together, genetically modified OP9 cells may be a better choice for co-culture system to induce hematopoietic differentiation from human ES cells. Future study will verify this notion.

\section{REFERENCES}

1) Amit M, Carpenter MK, Inokuma MS, Chiu CP, Harris CP, Waknitz MA, Itskovitz-Eldor J, Thomson JA: Clonally derived human embryonic stem cell lines maintain pluripotency and proliferative potential for prolonged periods of culture. Dev Biol 227: 271-278, 2000.

2) Bauer J, Herrmann F: Interleukin-6 in clinical medicine. Ann Hematol 62: 203-210, 1991.

3) Bhatia M, Bonnet D, Wu D, Murdoch B, Wrana J, Gallacher L, Dick JE: Bone morphogenetic proteins regulate the developmental program of human hematopoietic stem cells. J Exp Med 189: 1139-1148, 1999.

4) Broxmeyer HE: Biology of cord blood cells and future prospects for enhanced clinical benefit. Cytotherapy 7: 209-218, 2005.

5) Broxmeyer HE: The hematopoietic system: principles of therapy with hematopoietically active cytokines, $\mathrm{pp}$ 1-37. In Cytokines in the treatment of hematopoietic failure, Ganser A and Hoelzer D (Ed), Marcel Dekker, 
New York, 1999.

6) Broxmeyer HE: Myelosuppressive cytokines and peptides. pp 121-150. In Blood cell biochemistry, Vol 7. Hemopoietic growth factors, Whetton T and Gordon $\mathrm{T}$ (Ed), Kluwer Academic/Plenum Publishers, London, UK, 1996.

7) Chi L, Li Y, Stehno-Bittel L, Gao J, Morrison DC, Stechschulte DJ, Dileepan KN: Interleukin-6 production by endothelial cells via stimulation of proteaseactivated receptors is amplified by endotoxin and tumor necrosis factor-alpha. J Interferon Cytokine Res 21: 231-240, 2001.

8) Chu P, Lutzko C, Stewart AK, Dube ID: Retrovirusmediated gene transfer into human hematopoietic stem cells. J Mol Med 76: 184-192, 1998.

9) Dorshkind K: Regulation of hemopoiesis by bone marrow stromal cells and their products. Annu Rev Immunol 8: 111-137, 1990.

10) Eto K, Murphy R, Kerrigan SW, Bertoni A, Stuhlmann H, Nakano T, Leavitt AD, Shattil SJ: Megakaryocytes derived from embryonic stem cells implicate CalDAG-GEFI in integrin signaling. Proc Natl Acad Sci U S A 99: 12819-12824, 2002.

11) Hammacher A, Ward LD, Weinstock J, Treutlein $H$, Yasukawa K, Simpson RJ: Structure-function analysis of human IL-6: identification of two distinct regions that are important for receptor binding. Protein Sci 3: 2280-2293, 1994.

12) Hannum C, Culpepper J, Campbell D, McClanahan T, Zurawski S, Bazan JF, Kastelein R, Hudak S, Wagner J, Mattson J, et al: Ligand for FLT3/FLK2 receptor tyrosine kinase regulates growth of haematopoietic stem cells and is encoded by variant RNAs. Nature 368: 643-648, 1994.

13) Hirano T: The biology of interleukin-6. Chem Immunol 51: 153-180, 1992.

14) Itskovitz-Eldor J, Schuldiner M, Karsenti D, Eden A, Yanuka O, Amit M, Soreq H, Benvenisty N: Differentiation of human embryonic stem cells into embryoid bodies compromising the three embryonic germ layers. Molecular Medicine (Cambridge, Mass.) 6: 88-95, 2000.

15) Jacobsen SE, Okkenhaug C, Myklebust J, Veiby OP, Lyman SD: The FLT3 ligand potently and directly stimulates the growth and expansion of primitive murine bone marrow progenitor cells in vitro: synergistic interactions with interleukin (IL) 11, IL-12, and other hematopoietic growth factors. J Exp Med 181: 1357 $-1363,1995$.

16) Kee BL, Paige CJ: Murine B cell development: commitment and progression from multipotential progenitors to mature B lymphocytes. Int Rev Cytol 157: 129-179, 1995.

17) Kincade PW, Lee G, Pietrangeli CE, Hayashi S, Gimble JM: Cells and molecules that regulate B lymphopoiesis in bone marrow. Annu Rev Immunol 7: 111-143, 1989.

18) Kishimoto T, Akira S, Narazaki M, Taga T: Interleukin 6 family of cytokines and gp130. Blood 86: 1243-1254, 1995.

19) Ku H, Yonemura Y, Kaushansky K, Ogawa M: Thrombopoietin, the ligand for the $\mathrm{Mpl}$ receptor, synergizes with steel factor and other early acting cytokines in supporting proliferation of primitive hematopoietic progenitors of mice. Blood 87: 4544-4551, 1996.

20) Kurian KM, Watson CJ, Wyllie AH: Retroviral vectors. Mol Pathol 53: 173-176, 2000.

21) Le JM, Vilcek J: Interleukin 6: a multifunctional cytokine regulating immune reactions and the acute phase protein response. Lab Invest 61: 588-602, 1989.

22) McKenna HJ, Stocking KL, Miller RE, Brasel K, De Smedt T, Maraskovsky E, Maliszewski CR, Lynch DH, Smith J, Pulendran B, Roux ER, Teepe M, Lyman SD, Peschon JJ: Mice lacking flt3 ligand have deficient hematopoiesis affecting hematopoietic progenitor cells, dendritic cells, and natural killer cells. Blood 95: 3489-3497, 2000.

23) Nagasawa T: A chemokine, SDF-1/PBSF, and its receptor, CXC chemokine receptor 4 , as mediators of hematopoiesis. Int J Hematol 72: 408-411, 2000.

24) Nakano T, Kodama H, Honjo T: Generation of lymphohematopoietic cells from embryonic stem cells in culture. Science 265: 1098-1101, 1994.

25) Nakano T, Kodama H, Honjo T: In vitro development of primitive and definitive erythrocytes from different precursors. Science 272: 722-724, 1996.

26) Reubinoff BE, Pera MF, Fong CY, Trounson A, Bongso A: Embryonic stem cell lines from human 
blastocysts: somatic differentiation in vitro. Nat Biotechnol 18: 399-404, 2000.

27) Takahashi T, Yamada K, Tanaka T, Kumano K, Kurokawa M, Hirano N, Honda H, Chiba S, Tsuji K, Yazaki Y, Nakahata T, Hirai H: A potential molecular approach to ex vivo hematopoietic expansion with recombinant epidermal growth factor receptorexpressing adenovirus vector. Blood 91: 4509-4515, 1998.

28) Thomson JA, Itskovitz-Eldor J, Shapiro SS, Waknitz MA, Swiergiel JJ, Marshall VS, Jones JM: Embryonic stem cell lines derived from human blastocysts. Science 282: 1145-1147, 1998.

29) Trivedi P, Hematti P: Simultaneous generation of CD34(+) primitive hematopoietic cells and CD73(+) mesenchymal stem cells from human embryonic stem cells cocultured with murine OP9 stromal cells. Exp Hematol 35: 146-154, 2007.

30) Veiby OP, Jacobsen FW, Cui L, Lyman SD, Jacobsen
SE: The flt3 ligand promotes the survival of primitive hemopoietic progenitor cells with myeloid as well as B lymphoid potential. Suppression of apoptosis and counteraction by TNF-alpha and TGF-beta. J Immunol 157: 2953-2960, 1996.

31) Vodyanik MA, Bork JA, Thomson JA, Slukvin, II: Human embryonic stem cell-derived CD34+ cells: efficient production in the coculture with OP9 stromal cells and analysis of lymphohematopoietic potential. Blood 105: 617-626, 2005.

32) Vodyanik MA, Thomson JA, Slukvin, II: Leukosialin (CD43) defines hematopoietic progenitors in human embryonic stem cell differentiation cultures. Blood 108: 2095-2105, 2006.

33) Zsebo KM, Williams DA, Geissler EN, Broudy VC, Martin FH, Atkins HL, Hsu RY, Birkett NC, Okino KH, Murdock DC: Stem cell factor is encoded at the S1 locus of the mouse and is the ligand for the c-kit tyrosine kinase receptor. Cell 63: 213-224, 1990. 